\title{
OP-Technik und Erfahrungsbericht über den distalen AO-Femurnagel (DFN)
}

\author{
R. Grass, A. Biewener, K. Herzmann, H. Zwipp
}

\section{Zusammenfassung}

Golden-Standardversorgung extraartikulärer und kombiniert intra- und extraartikulärer distaler Femurfrakturen ist die Kondylenplattenosteosynthese $(\mathrm{CP})$, ein Verfahren, das im Vergleich zu intramedullären Retentionsverfahren nachweislich mit einer hohen Komplikationsrate einhergeht. Ein neu entwickelter intramedullärer Kraftträger zur Retention von supra- und supradiakondylären Femurfrakturen, der distale Femurnagel (DFN), wird vorgestellt. Es handelt sich um einen soliden unaufgebohrten Verriegelungsnagel aus Titan, der retrograd über die interkondyläre Notch implantiert wird und zwei distale Verriegelungsoptionen (zwei 6,0-mm-Verriegelungsschrauben oder eine 6,0-mm-Verriegelungsschraube und Spiralklinge) besitzt. Eigene vorab durchgeführte vergleichende biomechanische Testungen (DFN versus CP) zeigten für die axiale Hauptbeanspruchung eine überlegene Steifigkeit und Festigkeit des DFN gegenüber der Kondylenplatte und für osteoporotische Frakturen signifikante Stabilitätsvorteile der Spiralklingenverriegelung gegenüber der SchraubenverriegelungMethodik: Die klinischen Ergebnisse nach Osteosynthese distaler Femurfrakturen mit dem DFN wurden bei
73 Patienten mit 74 Frakturen (suprakondylären [AO-Klassifikation 33.AFrakturen], supra-diakondylären [33.C-Frakturen] und Femurschaftfrakturen [32.X-Frakturen]) im Rahmen einer Multizenterstudie prospektiv erfaßt. Ergebnisse: Implantation des Nagels und geschlossene Reposition der suprakondylären Fraktur sowie distale Verriegelung mittels Zielbügel wurden von den Operateuren überwiegend als leicht (91\%) eingestuft. Bei der Nachuntersuchung, die im Mittel nach 6 Monaten erfolgte, konnten 3 von 4 Patienten ihr Bein voll belasten und frei bewegen, wohingegen nur 3\% der Patienten über ständige Schmerzen klagten und nicht in der Lage waren, ihr Bein zu belasten. $5 \%$ wiesen ein Streckdefizit von mehr als $10 \mathrm{Grad}, 8 \%$ eine relevante Beinverkürzung von mehr als $15 \mathrm{~mm}$ und 15\% eine relevante Varus-/Valgus oder Rekurvationsfehlstellung der Femurschaftachse auf, bei $8 \%$ der Osteosynthesen kam es zu einem Auslockern der Verriegelungsschraube oder Spiralklinge. Alle Frakturen waren zum Zeitpunkt der Nachuntersuchung konsolidiert. Eine primäre oder sekundäre Spongiosaplastik war in keinem Fall notwendig geworden, eine Pseudarthrose wurde nicht gesehen. Je einmal wurde ein Osteosyntheseversagen, einmal ein Infekt und ein Nagelbruch be- obachtet. Das Behandlungsergebnis wurde von den Patienten rein subjektiv in $87 \%$ als sehr gut und gut und in $13 \%$ als befriedigend eingestuft. In der abschließenden Gesamtbeurteilung der verantwortlichen Operateure wurde der Verlauf in $84 \%$ als sehr gut und gut und in $17 \%$ als befriedigend eingeschätzt. Schlussfolgerung: Geschlossene Reposition und Retention von suprakondylären und supra-diakondylären Femurfrakturen sind in der beschriebenen Technik minimal invasiv und technisch einfach zu bewerkstelligen. Der DFN ist für die Versorgung extraartikulärer und kombiniert intraund extraartikulärer distaler Femurfrakturen ein geeignetes, sicheres und empfehlenswertes Implantat. Die Kombination größere Oberfläche der Spiralklinge mit daraus resultierender günstigerer Verteilung der auf den weichen metaphysären Knochen axial eingeleiteten Last und winkelstabile Verankerung lassen vor allem beim osteoporotischen Knochen und/oder hochgradig instabilen Frakturen bei der Spiralklingenverriegelung einen günstigen Einfluss auf das bei der Anwendung anderer distaler Nageltechniken beobachtete Problem der Bolzenlockerung, Bolzen- und Nagelprotrusion erwarten.

\section{Einleitung}

Golden-Standardversorgung extraartikulärer und kombiniert intra- und extraartikulärer distaler Femurfrakturen ist die Kondylenplattenosteosynthese, ein Verfahren, das nachweislich mit einer hohen Komplikationsrate einhergeht, wenn zur

OP-JOURNAL 2000; 16: 170-175

(C) Georg Thieme Verlag Stuttgart · New York
Erfüllung der Forderung nach einer anatomischen Reposition und stabilen Osteosynthese der suprakondyläre Frakturbereich offen reponiert wird $[3,8]$. Mechanische Stabilität und Vaskularität gelten unstrittig als die wichtigsten Faktoren der Bruchheilung, wobei für die Stabilität das Implantat und für die Durchblutung die Repositionstechnik ausschlaggebend sind [2].

Bei suprakondylären Mehrfragmentfrakturen ist das Potential für eine Übertra- gung der Kompressionskräfte von einem Fragment auf das andere herabgesetzt. Fehlt darüber hinaus medialseitig eine kortikale Abstützung, muss ein Implantat am distalen Femur alle entstehenden Kräfte neutralisieren. Bei idealem intramedullären Sitz und unter dynamischen Lastbedingungen wird ein Implantat am distalen Femur vorwiegend in axialer Richtung beansprucht. $\mathrm{Zu}$ dieser axialen Beanspruchung addiert sich ein Biegemoment, wenn der Kraftträger, wie dies für die Kondylenplatte der Fall ist, lateral der 
Last- und Schaftachse montiert ist. Als Instabilitätsfaktoren der Kondylenplatte gelten übereinstimmend deren laterale Position am Femur und daraus resultierendes zusätzliches Biegemoment sowie die winkelinstabile Platten-SchraubenVerankerung $[2,4]$. Als Ursachen für die angesprochene problematische Frakturheilung, wie sie nach Osteosynthese mit Kondylenplatte beschrieben wird $[3,8,10]$, kommt somit nicht nur die Beeinträchtigung der Vaskularität der Frakturzone durch den Standardzugang, sondern auch, und dies gilt vor allem für die Retention von instabilen Frakturen, eine ungeeignete Stabilität des Implantats in Betracht [4].

Weiss et al. [9] berichten über eine vergleichsweise verminderte Infekt- und Pseudarthrosenrate bei der retrograden Retention von suprakondylären Femurfrakturen mit dem Green-Seligson-Henry-Nagel (GSH-Nagel). Nageldesign (gerader kanülierter Verriegelungsnagel), Material (Stahl) sowie Implantationstechnik (aufgebohrter Nagel) des GSH-Nagels erscheinen biologisch ungünstig (die potentiell infektbegünstigenden Eigenschaften von Stahl, Kanülierung und Beeinträchtigung der intramedullären Durchblutung durch Aufbohren des Markraumes sind bekannt und wurden an anderer Stelle aufgezeigt $[1,7])$. Daneben beschreiben Weiss et al. in $8 \%$ der Fälle Probleme mit der distalen Verriegelung [9] (Bolzenlockerung und -protrusion).

Diese Erkenntnisse zugrundegelegt wurde auf Basis des unaufgebohrten Femurnagels (UFN) ein biomechanisch optimierter, unaufgebohrter, über das Kniegelenk retrograd zu implantierender Verriegelungsnagel aus Titan entwickelt [4] und entsprechend seiner Lokalisation am körperfernen Oberschenkel „distaler Femurnagel (DFN)“ genannt. Der Nagel wird im proximalen Schaftbereich standardmäßig mit zwei 4,9-mm-Bolzen und distal, optional mit zwei 6,0-mmSchrauben oder einer 6,0-mm-Schraube und einer rechtwinklig zur Nagelachse eingebrachten Spiralklinge verriegelt $[4,6]$.

Eigene vorab an Leichenfemura durchgeführte vergleichende biomechanische Testungen zeigten für die axiale Hauptbeanspruchung eine überlegene Steifigkeit und Festigkeit des DFN gegenüber der Kondylenplatte und für osteoporotische Frakturen signifikante Stabilitätsvorteile der Spiralklingenverriegelung gegenüber der Schraubenverriegelung $[4,5]$.

\section{Hauptteil}

\section{Op.-Technik für die Stabilisierung} extraartikulärer distaler

Femurfrakturen (AO-Klassifikation 33A-Frakturen) mit dem DFN

In Rückenlage wird auf einem röntgendurchlässigen Operationstisch vor Abwaschen und Abdecken das verletzte Kniegelenk durch Abkippen des Fußteils in eine $80^{\circ}$ Beugestellung gebracht und die unverletzte Extremität in einem Beinhalter gelagert. Unter Blutsperre (fakultativ) erfolgt ausgehend vom distalen Patellapol eine gerade $5 \mathrm{~cm}$ lange Inzision der Haut (Abb.1). Nach Durchtrennen des Subkutangewebes und Längsspalten des Ligamentum patellae wird der Hoffasche Fettkörper kranial von der Rückfläche des Ligamentum patellae abpräpariert und das Kniegelenk eröffnet. Nach Einsetzen von zwei Couley-Haken wird der First der interkondylären Notch aufgesucht. Zur besseren Darstellung kann dabei die Lichtquelle eines Arthroskops hilfreich sein.

Der kleine Finger der linken Hand wird an den First der interkondylären Notch geführt und stützt den 3,2-mm-Führungsdraht ab (357.129) (Abb.1), der im antero-posterioren und seitlichen Strahlengang (Abb.2) parallel zur Femurschaftachse, etwa $3 \mathrm{~mm}$ unterhalb und genau mittig in den First der interkondylären Notch (Abb.6) eingebracht wird.

Der medial von der Eintrittstelle liegende femorale Ansatz des hinteren Kreuzbandes wird dabei nicht tangiert (Abb.6).

Bei korrekter Positionierung, die mittels Bildwandler überprüft werden sollte, durchläuft der Draht im a.p.-Strahlengang die Kondylen in einem Winkel von $8^{\circ}$ zur Lotrechten der Kondylenebene.

Über den liegenden Führungsdraht wird die Gewebeschutzhülse (357.530) (Abb. 3,4) und der $13 \mathrm{~mm}$ kanülierte Spiralbohrer (351.270) (Abb.5) an die Notch herangeführt und die Kortikalis der interkondylären Notch eröffnet. Es genügt den Markraum zu eröffnen (Abb.6), ein Aufbohren des Markraums ist nicht erforderlich. Nach Entfernen von Bohrer und Führungsdraht wird der auf dem Zielbügel (357.112) (Abb.7) fixierte distale Femurnagel in den Markraum ein- und, unter Reposition der Fraktur, ins proximale Frakturfragment (Abb. 8) vorgebracht. Normalerweise gelingt das Einbringen des DFN manuell.

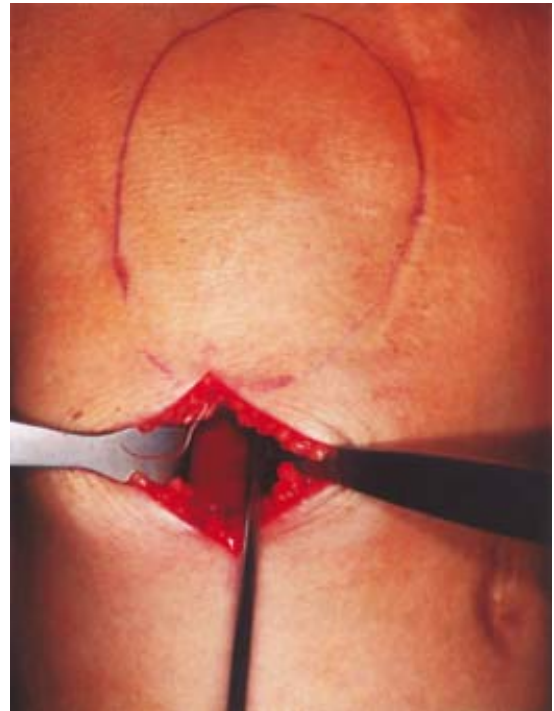

Abb.1 Zugangsweg und Lage des 3,2 mm Kirschner-Drahtes.

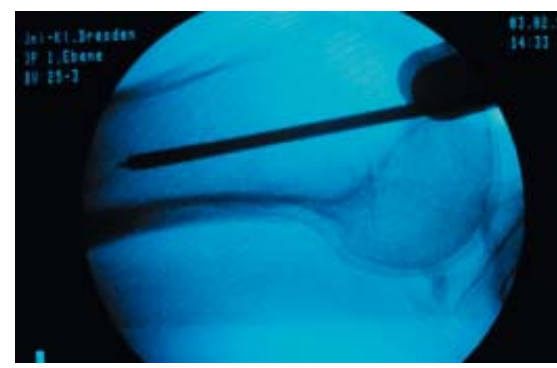

Abb. 2 Korrekte Lage des 3,2 mm KirschnerDrahtes im seitlichen Strahlengang.

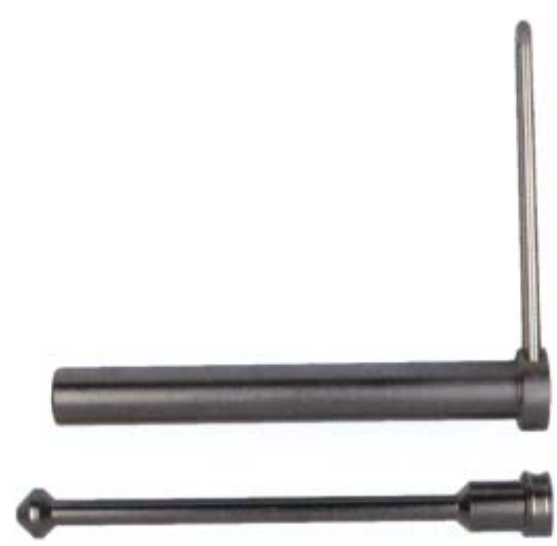

Abb.3 Gewebeschutzhülse des 13-mm-Spiralbohrers. 


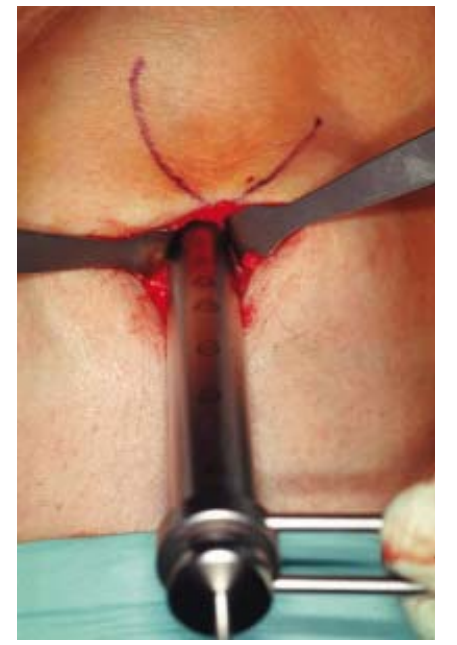

Abb. 4 Gewebeschutzhülse für 13-mm-Spiralbohrer in situ.

Abb. 5 Kanülierter $13 \mathrm{~mm}$ Spiralbohrer.

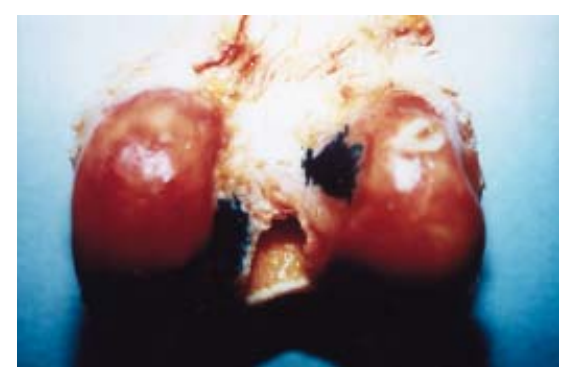

Abb. 6 Insertionspunkte der beiden Kreuzbänder und Lage der Nageleintrittsstelle interkondylär, von streckseitig gesehen.

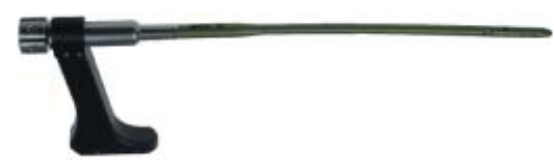

Abb. 7 Zielbügel und darauf fixierter DFN.

Bei jungen Patienten mit sehr harter Spongiosa kann es vorkommen, dass der DFN mit leichten Hammerschlägen vorgetrieben werden muss.

Nach endgültiger Platzierung des Nagels muss das distale Nagelende mindestens $2 \mathrm{~mm}$ innerhalb des subchondralen Knochens zum Liegen kommen. Da eine manuelle Bestimmung der Nageleinbringtiefe bei liegendem Zielbügel nicht gelingt, muss diese anhand der Ringmarkierungen (Abb. 8) des Zielbügelrohrs überprüft werden. Ein Nagelimpingement gehört zu den typischen Komplikationen der distalen Femurnagelung, aus diesem Grunde ist dieser Operationsschritt mit besonderer Sorgfalt durchzuführen.
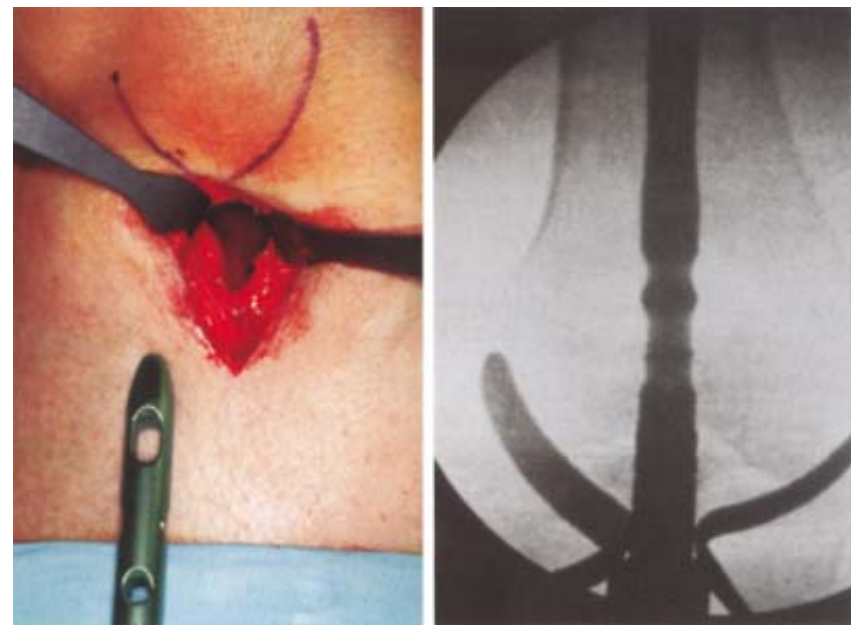

Abb. 8 DFN vor Implantation und Lage des DFN im a.p.-Strahlengang nach Implantation. Die Ringmarkierung (Pfeil) zeigt, dass der Nagel noch etwa $1,5 \mathrm{~cm}$ weiter vorgeschoben werden muss.
Entsprechend der Frakturmorphologie und/oder des Kalksalzgehaltes des Knochens, erfolgt die distale Verriegelung optional, entweder mit zwei 6,0-mm-Verriegelungsschrauben, oder der Spiralklinge und einer 6,0-mm-Verriegelungsschraube.

Für eine Standardverriegelung wird der Zielbügelaufsatz für Schraubenverriegelung auf den Zielbügel montiert und zunächst die proximale $6,0-\mathrm{mm}$-Verriegelungsschraube perkutan über Stichinzision (türkisfarbene Gewebeschutzhülse (357.118) (Abb.9) nach Aufbohren mit dem 4,9-mm-Spiralbohrer (357.099) plaziert.

Die Länge der zu verwendenden Schraube kann direkt auf dem 4,9-mm-Spiralbohrer abgelesen werden. Die zweite Schraubenverriegelung erfolgt über die kaudale Bohrung am Zielbügelaufsatz.

Erfolgt eine Verriegelung mit Spiralklinge, wird der Zielbügelaufsatz für Spiralklingenverriegelung (Abb. 10) (357.116) benutzt.

Auch bei dieser Methodik erfolgt zunächst das Einbringen der proximalen 6,0-mm-Verriegelungsschraube (s.o.) über das kraniale Verriegelungsloch am Zielbügelaufsatz. Über Stichinzision wird danach die rosafarbene zweifache Büchsenkombination (357.123) (Abb.11) an der lateralen Kortikalis aufgesetzt, über diese ein Führungsdraht bis in die Gegenkortikalis vorgebracht und, nach Bestimmen der zu verwendenden Spiralklingenlänge, die auf dem Führungsdraht abgelesen werden kann, mit dem 13,0-mm-Spiralbohrer nur die laterale Kortikalis eröffnet.

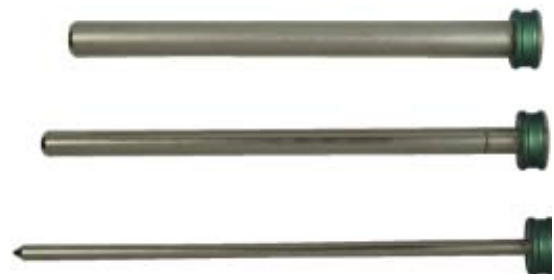

Abb.9 Gewebeschutzhülse für distale Schraubenverriegelung.

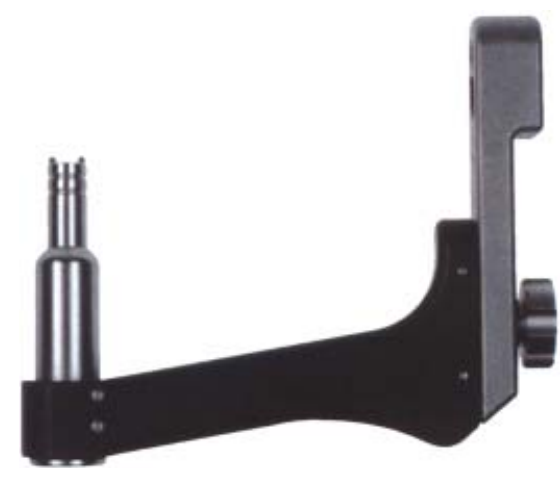

Abb.10 Zielbügelaufsatz für Spiralklingenverriegelung.

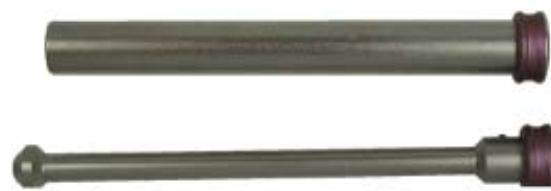

Abb.11 Gewebeschutzhülse für Spiralklingenverriegelung.

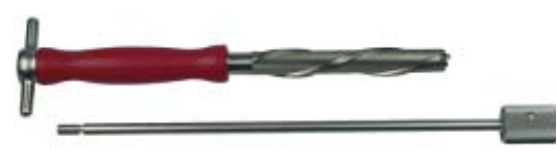

Abb.12 Spiralrohr. 


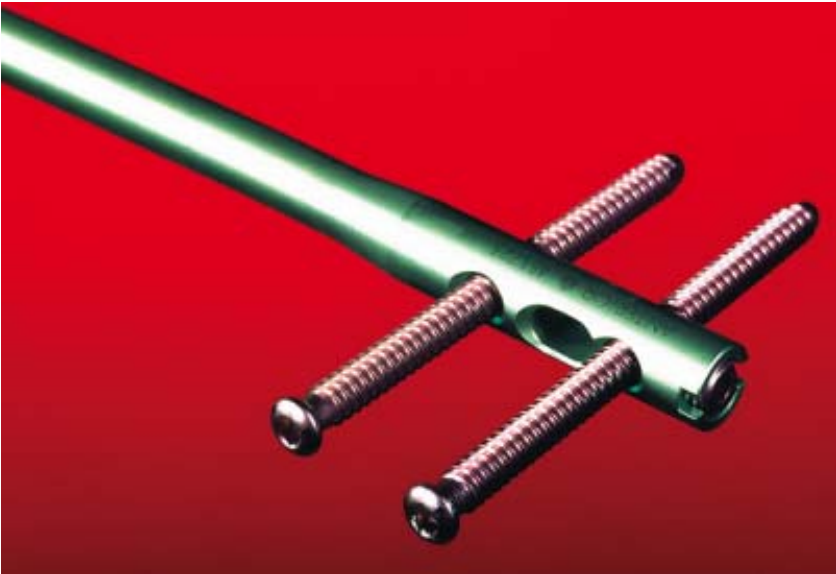

Abb.13 Distale Schraubenverriegelung.

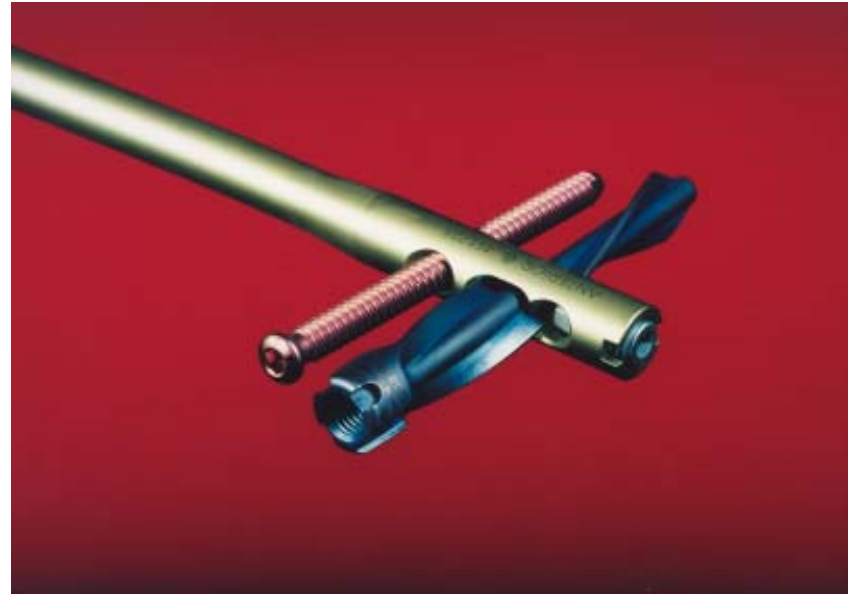

Abb.14 Distale Spiralklingenverriegelung.
Danach wird die auf dem Spiralrohr (357.120) (Abb.12) montierte Spiralklinge mit leichten Hammerschlägen so weit eingeschlagen, bis der Klingenkopf bündig mit der lateralen Kortikalis abschließt.

Die Spitze der Spiralklinge sollte dabei nicht die Gegenkortikalis überragen.

Durch axialen Zug am Zielbügel wird die Länge und Torsion des Femurs wiederhergestellt und, unter Beibehalten des Längszuges proximal, in Freihandtechnik (Bildwandler, röntgendurchlässiges Winkelgetriebe) standardmäßig mit zwei 4,9-mm-Bolzen verriegelt (Spiralbohrer $4,0 \mathrm{~mm}$ ). Die Verriegelung kann entsprechend der Frakturmorphologie statisch oder dynamisch erfolgen.

Bis zu einer Nagellänge von $240 \mathrm{~mm}$ empfiehlt sich ein latero-mediales, bei Nagellängen über $240 \mathrm{~mm}$ ein anteroposteriores Einbringen der 4,9-mm-Verriegelungsbolzen. Nach Entfernen des Zielbügels muss noch einmal manuell die Einbringtiefe des Nagels kontrolliert werden. Überragt der Nagel den subchondralen Knochen, wird daraus mit Sicherheit ein Impingement zwischen $\mathrm{Na}$ gelspitze und Patellarückfläche und entsprechender Beschwerdesymptomatik bei Beugung des Kniegelenkes über $100^{\circ}$ resultieren.

Entsprechend der distalen Verriegelung wird abschließend die Verschlussschraube (451.895/Schraube oder 451.896/Klinge) in das distale Nagelgewinde eingebracht und fest angezogen. Diese Schraube verklemmt die Spiralklinge bzw. die Verriegelungsschraube im Nagelschlitz (Abb.13,14), erhöht somit die Stabilität der Konstruktion und reduziert die Inzi- denz der Schrauben- bzw. Klingenlockerung.

Für eine einfache Fraktur ohne suprakondyläre Trümmerzone, distale Femurschaftfraktur und bei normalen Knochenverhältnissen ist eine Standardverriegelung (Abb. 13) ausreichend. Bei ausgedehnten suprakondylären Trümmerzonen und insbesondere bei pathologischen Frakturen (Metastasen), osteoporotischem Knochen wird eine Spiralklingenverriegelung (Abb. 14) empfohlen.

Nach ausgiebiger Spülung des Kniegelenkes erfolgt ein schichtweiser Wundverschluss unter Belassen einer intraartikulären Redondrainage ohne Sog, die am 2. postoperativen Tag entfernt wird.

\section{Op.Technik für die Stabilisierung} supra-diakondylärer Femurfrakturen (AO-Klassifikation 33C1-C3-Frakturen) mit offener Rekonstruktion der Kondylenfraktur

Liegt eine suprakondyläre Fraktur mit einfacher Gelenkfraktur (33.C1- oder 33.C2-Fraktur nach der AO-Klassifikation) vor, wird der Zugang modifiziert: Es wird entweder ein kleiner parapatellar medialer oder, falls die Hauptgelenkfraktur im Bereich der lateralen Kondyle liegt, ein kleiner parapatellar lateraler Zugang zum Kniegelenk gewählt. Nach Darstellen und Reposition der Gelenkfraktur wird diese temporär mittels Repositionszange und Kirschner-Drähten retiniert und definitiv mit Spongiosazugschrauben (6,5-mm-, kanülierte 7,3-mm-, oder KFI-Schrauben) retiniert.

Diese Schrauben sollten ventral platziert werden, damit sie nicht mit dem dorsal liegenden Nagel (Abb.15) interferieren.
Nach sicherer Retention der Gelenkfraktur kann die interkondyläre Notch in obengenannter Weise mit dem 13-mmSpiralbohrer oder, falls bei unklarer Stabilität der Kondylenosteosynthese Bedenken bestehen, in mm-Schritten eröffnet werden.

Diese letztgenannte Technik der NotchEröffnung sollte nach eigener Meinung bei der Versorgung von komplexen supra-diakondylären Frakturen (33C.3Frakturen nach der AO-Klassifikation) mit dem DFN immer zur Anwendung kommen.

Nach Einbringen des Nagels und proximaler Verriegelung mit einer 6,0-mmVerriegelungsschraube erfolgt dann optional, entsprechend der Frakturmorphologie eine distale Verriegelung mit Spiralklinge oder $6,0-\mathrm{mm}$-Verriegelungsschraube. Bei der Versorgung von 33C.1- und 33C.2-Frakturen erwies sich die Spiralklingenverriegelung bislang als unproblematisch, dennoch sollte, falls diese Verriegelungsoption gewählt wird, die Spiralklinge vorsichtig und unter Schutz der Gelenkfraktur durch eine Repositionszange eingebracht werden. Bei der Verriegelung von 33C.3-Frakturen ist in jedem Falle eine Standard-Schraubenverriegelung indiziert. Abschließend erfolgt intraoperativ eine Röntgenaufnahme des Oberschenkels mit Kniegelenk in 2 Ebenen mit einer Kassettenlänge von $60 \mathrm{~cm}$, die neben der Beurteilung der Frakturstellung auch eine Beurteilung der Femurachse gestattet.

\section{Nachbehandlung}

Unter Berücksichtigung der biomechanischen Erkenntnisse [4,5] und der bisheri- 


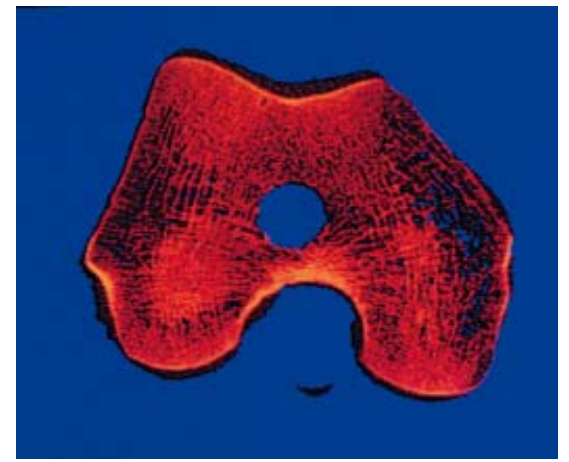

Abb.15 CT-Scan distaler Femur. Man beachte die dorsale Lage des Nagelkanals.

gen klinischen Anwendung des DFN [6] kann immer eine Freigabe des Kniegelenkes und eine funktionelle Nachbehandlung unter Teilbelastung mit $20 \mathrm{~kg}$ eingeleitet werden. Eine Röntgenkontrolle erfolgt nach Mobilisierung des Patienten in der ersten postoperativen Woche.

Zeigt sich in den weiteren Röntgenaufnahmen eine Kallusentwicklung (in der Regel ab der 3.-4. postoperativen Woche), kann die Belastung des Beines entsprechend der Schmerzsymptomatik unbedenklich gesteigert werden. Eine Vollbelastung des Beines sollte in der Regel nach 6-8 Wochen erreicht sein.

\section{Methodik}

Im Rahmen einer prospektiven Multizenterstudie [6] waren bei 73 Patienten (37 Männer und 36 Frauen mit einem Durchschnittsalter von 52 Jahren (17-96) mit 74 Frakturen (50\% 33.A-Frakturen, 33\% 33.CFrakturen und 17\% 32.XY-Frakturen) das operative Handling sowie die ersten klinischen Ergebnisse nach osteosynthetischer Versorgung distaler Femurfraktur mit dem DFN ermittelt worden. 33\% der Frakturen waren höhergradig offen (IOII 22\%, IOIII 11\%), und $32 \%$ der Patienten waren polytraumatisiert (durchschnittlicher IS-Score 28 Punkte). Im Mittel erfolgte die operative Versorgung 2,3 Tage (maximal 44 Tage) nach dem Unfall. Der Eingriff dauerte durchschnittlich 93 min (30-210) (bei 33.A- und Femurschaftfrakturen $80 \mathrm{~min}(30-180)$ und bei 33.C-Frakturen $120 \mathrm{~min}$ (45-210). Eine primäre oder sekundäre Spongiosaplastik erfolgte in keinem Fall. Gelegentlich wurde bei günstiger Frakturmorphologie frühpostoperativ eine Vollbelastung (17\%), in der Regel jedoch eine Teilbelastung (68\%) des Beines mit $20 \mathrm{~kg}$ gestattet und in $15 \%$ die Osteosynthese nur als übungsstabil eingestuft.

\section{Ergebnisse}

Implantation des Nagels und geschlossene Reposition der suprakondylären Fraktur sowie distale Verriegelung mittels Ziehbügel wurden von den Operateuren überwiegend als leicht ( $91 \%$ ), bei den ersten Fällen ausnahmsweise als schwierig eingestuft. Zum Zeitpunkt der Nachuntersuchung, im Mittel 6 (4-15) Monaten nach der Operation, konnten 3 von 4 Patienten ihr Bein voll belasten und frei bewegen, wohingegen nur $3 \%$ der Patienten über ständige Schmerzen klagten und nicht in der Lage waren, ihr Bein zu belasten. Alle Frakturen waren zum Zeitpunkt der Nachuntersuchung konsolidiert oder in Konsolidierung begriffen.

4 Patienten (5\%) wiesen ein Streckdefizit von mehr als 10 Grad, 6 Patienten (8\%) eine relevante Beinverkürzung von mehr als $15 \mathrm{~mm}$ und 11 Patienten (15\%) eine relevante Varus-/Valgus-(Fehlstellungswinkel größer als 5 Grad) oder Rekurvationsfehlstellung (Fehlstellungswinkel größer als 10 Grad) der Femurschaftachse auf. Ein sekundärer Verlust des primär erreichten Repositionsergebnisses wurde einmal, bei einer 93-jährigen Patientin nach operativer Versorgung einer 33.A2-Fraktur beobachtet: Im Rahmen einer unkontrollierten Vollbelastung war am 3.postoperativen Tag der Nagel ausgebrochen.

Bei $8 \%$ der Osteosynthesen wurde ein Auslockern, entweder der Spiralklinge oder der proximalen 6,0-mm-Verriegelungsschraube beobachtet. Einmal brach der DFN auf Höhe eines proximalen Bolzenlochs. Einmal kam es bei einer multimorbiden hochbetagten Patientin zu einem Infekt, der nach insgesamt 3 Revisionseingriffen und Verfahrenswechsel auf DCS inzwischen ausgeheilt ist. 2 Patienten mussten wegen eines primär nicht genügend weit vorgebrachten Nagels, dessen Spitze bei der Kniegelenksbeugung mit der retropatellaren Gelenkfläche interferierte (nach röntgenologischen Kriterien musste die Nageleinbringtiefe in 18\% als nicht ausreichend eingestuft werden) reoperiert und der Nagel weiter nach proximal vorgebracht werden. Bei den 33C-Frakturen (33\%) kam es durch Einbringen des DFN bzw. der Spiralklinge in keinem Fall zu einer Dislokation der zuvor mit Schrauben retinierten artikulären Fraktur.

Das Behandlungsergebnis (Abb.16,17) insgesamt wurde von den Patienten in $39 \%$ als sehr gut, in $48 \%$ als gut und in

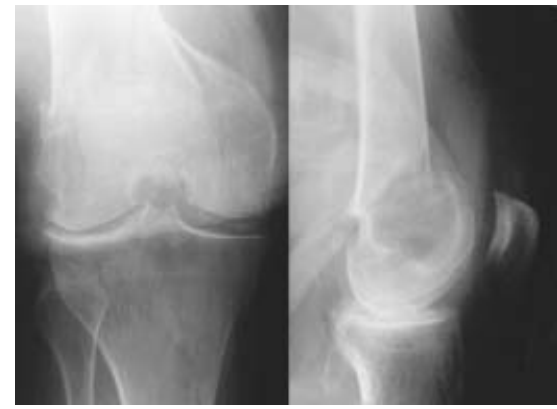

Abb.16 86-jährige mit einer 33C2.1-Fraktur.

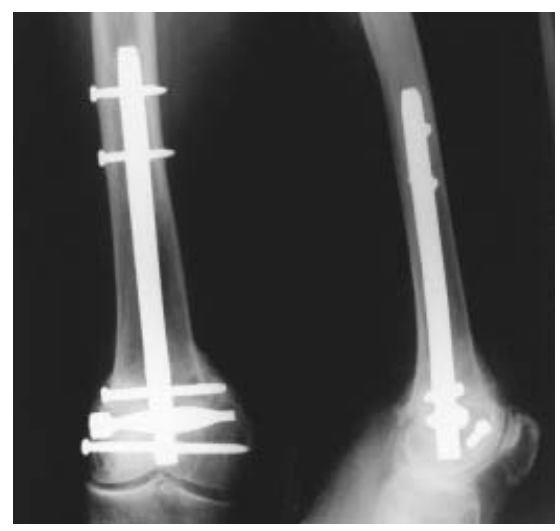

Abb.17 Gleiche Patientin nach Ausheilung der Fraktur in der 16. postoperativen Woche.

$13 \%$ als befriedigend eingestuft. Diese subjektive Einschätzung deckt sich mit der abschließenden Gesamtbeurteilung der verantwortlichen Operateure, die das Behandlungsergebnis in $43 \%$ als sehr gut, in $41 \%$ als gut und in $17 \%$ als befriedigend einstuften.

\section{Diskussion}

Vaskularität und geeignete mechanische Stabilität gelten als die beiden wichtigsten Faktoren für die Knochenheilung; Repositionstechnik und Wahl des Implantates stellen somit die Weichen für die Frakturheilung. Bei den in der Studie [6] erfassten Patienten erfolgte immer eine die Vaskularität respektierende, geschlossene Reposition der suprakondylären Frakturzone sowie Retention mit einem in vitro erwiesenermaßen biomechanisch stabilen Implantat. Minimal traumatisierendes Vorgehen und Retention der Fraktur durch ein ,bio-logisch optimiertes und, im In-vitro-Vergleich mit der Kondylenplatte, stabilerem Implantat hatten den erwartetet günstigen Einfluss auf Infekt- und Pseudarthrosenrate. Auch schwere Bruchformen heilten ohne Durchführung einer primären oder sekundären Spongiosaplastik in 96\% der Behandlungen unproblematisch 
aus. Diese Ergebnisse kontrastieren mit den Resultaten nach GSH-Nagel- sowie konventioneller und ,bio-logischer' Kondylenplattenosteosynthese [10], bei denen Infektraten bis zu 7\% [3], Pseudarthrosenraten bis zu 8\% [8], beim Golden-Standardverfahren ein Anteil von primären oder sekundären Spongiosaplastiken bis zu 21\% [3], bei ,biologischer“ Kondylenplattenosteosynthese von bis zu 20\% [10] und nach GSH-Nagelosteosynthese [9] von bis zu $8 \%$ beschrieben werden.

Eine relevante Varus- oder Valgusfehlstellung oder Ante-Rekurvationsfehlstellung wurde bei insgesamt $15 \%$ der Osteosynthesen gesehen. Diese nicht unerhebliche Inzidenz von relevanten Achsfehlstellungen des Femurs nach DFN-Osteosynthese erklärt sich mit der geschlossenen Repositionstechnik und gibt zu denken. Probleme mit der korrekten Achsausrichtung des distalen Femurs werden auch bei Anwendung der Golden-Standardtechnik beschrieben [11]. So finden Zehnter et al. [11] nach Kondylenplattenosteosynthese identische Winkelwerte für die Femurschaftachse beider Beine bei nur 24\% der Patienten und in 26\% relevante Varus-/Valgus- sowie in $22 \%$ relevante Ante- bzw. Rekurvationsfehlstellungen. Die Ergebnisse beider Studien sind nicht zufriedenstellend, die geringere Inzidenz an relevanten Achsfehlern nach DFN-Osteosynthese läßt jedoch vermuten, dass es über die intramedulläre Repositions- und Retentionsmethodik leichter gelingt die anatomische Achse des Femurs wiederherzustellen, von einer , automatischen Reposition' kann jedoch keine Rede sein. An einer Methodik intraoperativ eine korrekte Achsausrichtung des distalen Femurs zu erreichen, wird derzeit gearbeitet. Bei problematischen Frakturen kann der Fixateur externe als temporäres Reduktionsverfahren angewendet werden.

\section{Schlussfolgerung}

Die klinischen Resultate ergänzen und bestätigen die in vitro gewonnenen biomechanischen Ergebnisse und lassen für die DFN-Osteosynthese folgende vorläufige Schlussfolgerungen zu:

Geschlossene Reposition und Retention von suprakondylären und supra-diakondylären Femurfrakturen sind in der beschriebenen Technik minimal invasiv und technisch einfach zu bewerkstelligen.

Die größere Oberfläche der Spiralklinge erlaubt eine günstigere Verteilung der auf den weichen metaphysären Knochen axial eingeleiteten Last. Bessere Lastverteilung und stabilere Verankerung der Spiralklinge durch Verschlussschraube lassen vor allem beim osteoporotischen Knochen und/oder hochgradig instabilen Frakturen, mit langstreckigen suprakondylären Trümmerzonen, einen günstigen Einfluss auf das bei der Anwendung anderer distaler Nageltechniken beobachtete Problem der Bolzenlockerung, Bolzenund Nagelprotrusion erwarten.

Der distale Femurnagel ist für die Versorgung extraartikulärer und kombiniert intra- und extraartikulärer distaler Femurfrakturen ein geeignetes, sicheres und empfehlenswertes Implantat. Der DFN kann, die ersten klinischen Ergebnisse zugrundegelegt, zudem als sichere Alternative zum unaufgebohrten Femurnagel (UFN) bei folgenden Indikationen empfohlen und angewendet werden:

- Femurschaft- und gleichseitige Patellafraktur,

- Femurschaft- und gleichseitige Tibiafraktur,

- Femurschaft- bei gleichseitiger Acetabulumfraktur,

- Femurschaftfraktur bei ausgeprägter Adipositas.

\section{Literatur}

${ }^{1}$ Arens S, Schlegel U, Printzen G, Perren S, Hansis M. Einfluss des Implantatmaterials auf die lokale Infektentstehung. Tierexperimentelle Untersuchung von DC-Platten aus V4A-Stahl und Reintitan. DGU-Tagung Abstract-Band 1995; 59

${ }^{2}$ Baumgaertel F, Perren SM, Rahn B. Tierexperimentelle Untersuchungen zur „biologischen“ Plattenosteosynthese von Mehrfragmentfrakturen des Femurs. Unfallchirurg 1994; 97: 19-27

${ }^{3}$ Haas N, Schandelmaier P, Krettek C. Therapeutisches Konzept bei der distalen Femurfraktur mit Gelenkbeteiligung. Hefte zur Unfallheilkunde, Springer Verlag 1990; 212: 179-187

${ }^{4}$ Ito K, Grass R, Zwipp H. Internal Fixation of Supracondylar Femoral Fractures: Comparative Biomechanical Performance of the 95Degree Blade Plate and Two Retrograde Nails. J Orthop Trauma, 1998; Vol. 12: No.4

${ }^{5}$ Ito K, Hungerbühler R, Wahl D, Grass R. Improved Intramedullary Nail Interlocking in Osteoporotic Bone. J Orthop Trauma (im Druck)

${ }^{6}$ Grass R, Biewener A, Herzmann K, Wagner R, Zwipp H. Operative Versorgung distaler Femurfrakturen mit dem distalen Femurnagel (DFN) - Eine prospektive Studie. Acta chirg austr. (im Druck).

${ }^{7}$ Melcher GA, Claudi B, Schlegel U, Perren S, Printzen M, Munzinger G. J. Influence of Type of Medullary Nail on the Development of Local Infection. J. Bone Joint Surg. 1994; 76 B: $955-959$

${ }^{8}$ Tscherne H, Oestern H, Trentz, O. Long Term Results of the Distal Femoral Fractures and its Special Problems. Zentralbl Chir 1977; 102 (15): 897-904

${ }^{9}$ Weiss G, Seligson D, Wurst Ch. The Intramedullary Supracondylar Nail. The first One Hundred Cases. Kongressbericht Osteosynthese International 1994. P. M. Rommens/ V. Vecsei. Leuven University Press 1995; $46-48$

${ }^{10}$ Wenda K, Runkel M, Rudig L. Die „durchgeschobene" Kondylenplatte. Unfallchirurgie 1995; 21: 77-82

${ }^{11}$ Zehntner M, Marchesi D, Burch H, Ganz R. Alignment of Supracondylar/Intercondylar Fractures of the Femur after Internal Fixation by AO/ASIF Technique. J Orthop Trauma 1992; 6 (3): $318-326$

Dr. med. R. Grass

Oberarzt

A. Biewener

Assistenzarzt

K. Herzmann

Assistenzarzt

Klinik für Unfall- und Wieder-

herstellungschirurgie der TU Dresden

Prof. Dr. med. H. Zwipp

Ärztlicher Direktor

Universitätsklinikum C. G. Carus

Fetscherstr. 74

01307 Dresden 